\title{
The impact of the intracerebral antibody response on the clinical course of a virus-induced demyelination in a rat model system
}

\author{
R Dörries, H Imrich, A Hein, S Czub, S Schwender
}

The repertoire of tools for therapeutic intervention in demyelinating disorders of the CNS, such as multiple sclerosis or postinfectious encephalomyelitis, is still very limited. This deficit is mainly because the pathogenesis of these serious complications is far from being completely understood. In this context, animal models of virus-induced demyelinating encephalomyelitis are gaining in importance as a promising platform for the development of therapeutic strategies. Among these model systems intracerebral infection of rodents by the murine coronavirus JHM (JHMV) is particularly interesting. ${ }^{1}$ We review our current data on the role of the humoral immune system response in the clinical course of JHMV infections in rat inbred strains.

Course of the disease and histopathology In Lewis rats, JHMV can trigger a monophasic neurological disease when given intracerebrally at the age of three weeks. ${ }^{2}$ Typically, a rapid onset of such symptoms as, hind leg paresis and ataxic gait are seen approximately one week after infection. Severity of symptoms increase up to day 12 after infection and some animals may have tetraplegia. At this stage $40 \%$ of the animals have usually succumbed to acute encephalitis. The remainder recover from disease and four weeks after infection almost no overt signs of the infection remain. Histopathologically, the disease is accompanied by multiple areas of vacuolising demyelination in the brain. Swollen axons can be detected and affected areas are infiltrated by numerous mononuclear cells that most likely travel from frequent perivascular cuffs to the affected areas.

\section{The inflammatory reaction in the CNS}

To identify the lymphocyte subsets that home to demyelinated areas, a combination of immunohistology and computer aided cell morphometry was developed. Animals were infected intracerebrally with JHMV and killed 7, 14 and 21 days after infection. After thorough perfusion of the animals with phosphate buffered saline, the entire CNS including the spinal cord was snap frozen in liquid nitrogen. Subsequently, serial frozen sections of $6 \mu \mathrm{m}$ were cut from the CNS tissue and indirect immune alkaline phosphatase staining was used to detect viral antigens, $\mathrm{CD}^{+}$cytotoxic $\mathrm{T}$ lymphocytes, $\mathrm{CD}^{+}{ }^{+}$helper $\mathrm{T}$ lymphocytes, and immunoglobulin (Ig) containing B lymphocytes. ${ }^{2}$ Pictures of these stained sections were digitised by a video camera mounted on the microscope and stored in a computer. Subsequently, individual pictures were recalled to the screen and the red colour of the immune alkaline phosphatase staining was changed to any desired colour. Finally, merging of all pictures of a single virus-infected site resulted in a pseudocoloured topographical map of lymphocyte subset arrangements in relation to virus-infected target cells.

Seven days after infection $\mathrm{CD} 8^{+} \mathrm{T}$ lymphocytes are the dominating lymphocyte population focusing in virus-infected areas. They are in close contact with the viral target cell and only occasionally $\mathrm{CD}^{+}$helper $\mathrm{T}$ cells are scattered in the area. Most important, B lymphocytes are virtually absent at that time past infection. This picture dramatically changes at 14 days after infection, when animals enter convalescence. Multiple clusters of B lymphocytes are detectable in virus-infected areas that are surrounded by numerous $\mathrm{CD}^{+}{ }^{+} \mathrm{T}$ helper cells. These B cells are fully differentiated plasma cells because they stain intensely for intracellular Ig, but not for the B-cell specific isoform of the leukocyte common antigen detected by the monoclonal antibody OX $33 .{ }^{3}$ At 21 days after infection, most of the virus has been cleared from the CNS and residual virus-infected areas are infiltrated predominantly by $\mathrm{CD}^{+} \mathrm{T}$ lymphocytes accompanied by Ig-containing plasma cells and less $\mathrm{CD}^{+} \mathrm{T}$ cells. Additional stainings with the monoclonal antibody ED1, ${ }^{4}$ that detects rat macrophages, revealed that macrophages outnumber by far any other leukocyte population in the demyelinated plaques. Previous observations made by Watanabe et $a l,{ }^{5}$ have shown that these macrophages are filled with myelin debris, indicating their role as a scavenger cell in the disease process.

These data clearly indicate that the composition of leukocytes infiltrating virus-affected areas of the CNS changes significantly in the course of JHMV infection. To examine the dynamics of this process, we isolated leukocytes from the entire CNS of infected rats by density Percoll gradient every second day after infection and characterised them phenotypically by subsequent flow cytometry. ${ }^{2}$ At the onset of clinical symptoms, lymphocytes start to accumulate in the tissue. Their amount peaks with the maximum of neurological symptoms and with convalescence their level 
gradually drops back to that seen before the outbreak of the disease. $T$ lymphocytes $\left(\mathrm{CD}^{+}\right.$as well as $\left.\mathrm{CD}^{+}\right)$are the dominant populations constituting more than $90 \%$ of all recoverable lymphocytes. The quantity of $\mathrm{B}$ lymphocytes is almost negligible over the whole observation period. Nevertheless, at the beginning of convalescence their amount increases slightly. This is exactly at the time when Ig-containing plasma cells can be detected immunohistologically in the infected plaques.

\section{Intracerebral antibody secretion}

Examination of CNS-isolated leukocytes for virus-specific antibody secretion on a single cell base by ELISpot assay ${ }^{6}$ disclosed that the slight increase of B lymphocytes in CNS tissue was of tremendous importance for the outcome of the infection. ${ }^{7}$ Coincidentally with the small peak of B cells, a considerable quantity of virus-specific IgG secreting plasma cells appears in the brain tissue. Synchronously, virus-neutralising titres increase in CSF specimens and recovery of animals starts when a critical threshold of antibody secreting plasma cells is reached (table), suggesting that the humoral virus-specific immune system response could be essential for recovery from disease. This idea was further substantiated by results from the same assay conducted in rats of the Brown Norway inbred strain after infection with JHMV. ${ }^{7}$ Brown Norway rats are clinically completely resistant to JHMVinduced encephalomyelitis. ${ }^{5}$ At the tissue level, only a few, small virus-affected areas can be detected that are regularly situated in clinically silent periventricular sites and infiltrated by large plasma cells. Isolation of leukocytes from CNS tissue, flow cytometric quantitation and ELISpot assay revealed that the contribution of virus-specific antibody secreting plasma cells to the total of B cells in the brain parenchyma is much higher in Brown Norway rats compared with Lewis rats. This indicates that differentiation of $\mathbf{B}$ cells into antibody secretors is more effective and specific in the Brown Norway rat strain. Consequently, the virus neutralising antibody titre rises earlier and much more rapid in the CSF of Brown Norway rats (table). These data verified earlier observations from our laboratory, where we demonstrated a strong polyclonal intrathecal antibody synthesis with specificity for the virus in CSF specimens of Brown Norway rats, ${ }^{8}$ whereas intrathecally

The intracerebral virus-specific antibody response in Lewis and Brown Norway rats after infection with coronavirus $\mathfrak{F H M}$

\begin{tabular}{lccc}
\hline Rat strain & Days after infection & AbSC (SE) & $N T(S E)$ \\
\hline \multirow{3}{*}{ Lewis } & 7 & 0 & 0 \\
& 14 & $377(83)$ & $21(2)$ \\
Brown Norway & 20 & $320(428)$ & $17(1)$ \\
& 7 & $56(22)$ & $22(4)$ \\
& 14 & $2828(632)$ & $102(2)$ \\
& 20 & $553(217)$ & $83(3)$ \\
\hline
\end{tabular}

AbSC = virus specific IgG secreting cells extracted from the entire CNS; NT = JHMV neutralising antibody titre as determined in cerebrospinal fluid specimens; $\mathrm{SE}=$ standard error; the average of AbSC and NT was calculated from three animals/day. synthesised immunoglobulins in Lewis rats were more often of unknown specificity. ${ }^{9}$

\section{Role of B lymphocytes in the clinical course of the infection}

Given that efficient restriction of extracellular viral spread by $B$ cells is a prerequisite for a subclinical course of the infection, a complete absence of the B lymphocyte compartment should result in a disastrous clinical course of the infection. This hypothesis is strengthened by yet unpublished experiments from our laboratory in $\gamma$-irradiated Lewis rats. The immune system of Lewis rats was completely destroyed by sublethal $\gamma$-irradiation and 24 hours later they were infected with JHMV. The average of neurological symptoms was recorded over the next nine days.

Irradiation of animals and complete reconstitution before infection by transfer of spleen and lymph node cells from naive donors resulted in an almost identical development of disease as seen in non manipulated rats. However, when animals were irradiated and reconstituted by $\mathrm{CD}^{+}$and or $\mathrm{CD}^{+} \mathrm{T}$ lymphocytes in the absence of $B$ cells, the onset of disease was much earlier and progression to a moribund state was more rapid and frequent, compared with the fully competent animals. This is not only indicative of the essential role of B lymphocytes in protecting from severe neurological disease but suggests also that the action of $T$ cells in the absence of a virus-specific antibody response is of a pathogenic nature.

\section{Conclusion}

We think that in this model the rapid recruitment of a virus-specific antibody response to the brain tissue as seen in the Brown Norway rats will efficiently limit viral spread in the tissue. As a result only a few tissue damaging $T$ effector cells are sufficient to clear the virus on a subclinical level. Any delay in this process as seen in the Lewis rat, allows wide-spread dissemination of the virus in the white matter. As a result, inflammatory $T$ cells are attracted that will damage tissue as long as the formation of new secondary viral foci is not interrupted by virus-specific antibody secretion. In the absence of a functional B cell compartment this process will eventually cause death. However, for complete elimination of the virus from the brain, antibodies alone are not sufficient. It is the combined and rapid action of the humoral and cellular branch of the immune system that characterises a recovery from virus-induced demyelinating encephalomyelitis. This work was supported by the Bundesminister für Marion Zips, Ursel Sauer and Christine Kugler for expert technical assistance.

1 Dörries R. Background paper-On the role of the immune response in the course of coronavirus JHM-induced encephalomyelitides in mice and rats. Adv Exp Med Biol 1990;276:623-7. 
2 Dörries $\mathrm{R}$, Schwender S, Imrich $\mathrm{H}$, et al. Population dynamics of lymphocyte subsets in the CNS of rats with different susceptibility to coronavirus-induced demyelinating encephalitis. Immunology 1991;74:539-45.

3 Woollett GR, Barclay AN, Puklavec M, et al. Molecular and antigenic heterogeneity of the rat leucocyte-common antigen from thymocytes and $T$ and $B$ lymphocytes. Eur F Immunol 1985;15:168-73.

4 Dijkstra CD, Döpp EA, Joling P, et al. The heterogeneity of mononuclear phagocytes in lymphoid organs: Distinct macrophage subpopulations in the rat recognized by monoclonal antibodies ED1, ED2 and ED3. Immunolog 1985;54:589-99.

5 Watanabe $\mathrm{R}$, Wege $\mathrm{H}$, ter Meulen $\mathrm{V}$. Comparative analysis of coronavirus JHM-induced demyelinating encephalomyelitis in Lewis and Brown Norway rats. Lab Invest myelitis in Lew

6 Sedgwick J, Cook A, Dörries $R$, et al. Detection and enumeration of single antibody- or cytokine secreting cells
by the ELISA-plaque (ELISPOT) assay. In: Zola H, ed. Laboratory Methods in Immunology, vol I. Boca Raton, Laboratory Methods in

7 Schwender S, Imrich H, Dörries R. The pathogenic role of virus-specific antibody secreting cells in the central nervous system of rats with different susceptibility to coronavirus-induced demyelinating encephalitis. Immunology 1991;74:533-8.

8 Dörries $\mathrm{R}$, Watanabe $\mathrm{R}$, Wege $\mathrm{H}$, et al. Analysis of the intrathecal humoral immune response in Brown Norway (BN) rats, infected with the murine coronavirus JHM. Neuroimmunol 1987;14:305-16.

9 Dörries $\mathrm{R}$, Watanabe R, Wege $\mathrm{H}$, et al. Murine coronavirus induced encephalomyelitides in rats: Analysis of immunoglobulins and virus-specific antibodies in serum and cerebrospinal fluid. $₹$ Neuroimmunol 1986;12: 131-42. 